\title{
Étude de la Cinétique d'adsorption des molécules anti paludéennes par les résines
}

\author{
Nikita Topanou ${ }^{1 *}$, Biaou Dimon 1, 2, Taofiki Aminou1 \\ 'Laboratoire d'Expertise et de Recherche en Chimie de l'Eau et de l'Environnement, Université d'Abomey-Calavi, \\ Bénin \\ ${ }^{2}$ Centre Béninois de Recherche Scientifique et Technique, Université d'Abomey-Calavi, Bénin \\ Auteur Correspondant email: Nikita Topanou tnikit@yahoo.fr \\ Original submitted in on 30 th $A$ pril 2013 Published online at www.m.elewa.org on $29^{\text {th }}$ May 2013. \\ http://dx.doi.org/10.4314/jab.v65i0.89640
}

\section{RÉSUMÉ}

Objectif : Cet article présente les résultats expérimentaux de l'adsorption statique et dynamique des molécules de sulfate de chloroquine par la résine DOWEX $50 \mathrm{~W}$, avec pour objectif de proposer une technique en vue d'épurer le sang de certains médicaments contenus en surdose dans l'organisme., Méthodologie: La méthode utilisée est une modélisation in vitro de l'extraction des molécules médicamenteuses du sang par une adsorption dynamique ; l'adsorption statique simule l'extraction des molécules médicamenteuses de l'estomac.

Des essais d'adsorption par différents résines afin de déterminer celles qui adsorbent le mieux la chloroquine ont été effectués. L'influence des différents paramètres (Masse de l'adsorbant, concentration initiale, température, $\mathrm{pH}$, débit) sur le pouvoir adsorbant des résines a été également étudiée.

Résultats: L'adsorption cinétique des médicaments dépend de l'équilibre entre la fraction adsorbée et la fraction libre en solution. L'influence de la concentration initiale de la molécule, de la masse de résine, de la température et du $\mathrm{pH}$ de la solution a été évaluée. Les différents résultats obtenus, dans la modélisation in vitro permettent de confirmer une plausible influence de la masse de résine dans l'élimination des médicaments du sang, de l'estomac et du duodénum.

Mots Clé : modélisation, adsorption dynamique, sulfate de Chloroquine, résine DOWEX 50W, Constante d'équilibre

\begin{abstract}
Objectives: This paper presents experimental results of static and dynamic adsorption of molecules of chloroquine sulfate by DOWEX $50 \mathrm{~W}$, with the aim of proposing a technique to purify the blood content of certain drugs overdose in the body.

Methodology: This study is a model of in vitro experiments. The dynamic adsorption simulates the elimination of drugs from blood, and the static adsorption simulates the elimination of drugs from stomach. Chloroquine adsorption tests were conducted by different resins to determine which has the best adsorptive as regards chloroquine. The influence of different parameters (mass of adsorbent, initial concentration, temperature, $\mathrm{pH}$, flow rate) on the adsorptive resins was also studied.
\end{abstract}

\footnotetext{
*Auteur correspondant : Nikita TOPANOU: tnikit@yahoo.fr
} 
Results: The adsorption Kinetics of this drug depends on the balance between adsorbed and free substance in the solution. The influences of initial concentration $(\mathrm{Co})$ of the drug, the mass of the resin, the temperature and the $\mathrm{pH}$ of the solution have been evaluated. The quantities of adsorbed chloroquine sulfate decreases. The different results obtained, in this in vitro modeling, can confirm the probable role of the resin in the elimination of a drug from blood, stomach or duodenum.

Key words: modelisation, dynamic adsorption; chloroquine sulfate; resin DOWEX 50W; Kinetic constant.

\section{INTRODUCTION}

Les résines poreuses macromoléculaires ont été largement utilisées pour l'évaluation de la concentration de composés organiques à l'état de traces dans l'eau. Ceci est particulièrement vrai pour les résines polystyrène divinylbenzène de type XAD-2 et XAD-4. En plus de l'analyse chimique des pesticides et d'autres traces organiques, l'adsorption sur les résines XAD est devenue une des méthodes standards pour la préparation d'extraits relatifs aux analyses bactériologiques (Xiaomin et al., 2013). La première étude complète de l'utilisation de la résine $X A D-2$, qui remonte en 1974, a prouvé que l'adsorption sur XAD-2 était une méthode intéressante pour concentrer, sur la résine, un grand nombre de composé de concentrations comprises entre 0,2 et $50.000 \mathrm{PPb}$ (Shujuan et al., 2012). La résine $X A D-2$ est fréquemment utilisée pour concentrer les substances humiques contenues dans l'eau de mer. Plus récemment Karamanis and Vardoulakis (2012) ont montré que l'adsorption sur XAD-2 est une technique envisageable pour l'analyse de polluants isolés dans les eaux usées industrielles.

Cependant, plusieurs rapports critiques présentant les limites de la méthode ont aussi été publiés. En effet, l'impureté de la résine et le faible recouvrement entraînent une adsorption et une désorption incomplète. Ainsi, la technique d'adsorption sur XAD-2 n'est pas souhaitable pour la détection et la quantification des composés organiques en dessous du PPb (kunquan et al., 2012). Par ailleurs, même l'extraction d'une substance, d'une solution constitue une méthode de purification qui est mise en œuvre tant au niveau de l'industrie que de la recherche (Bouhadiba et al., 2003; Xiaomin et al., 2013).

L'adsorption par des résines constitue un procédé intéressant de purification et d'isolement; ce processus est utilisé dans l'hémoperfusion. Cette dernière consiste en une circulation extracorporelle dont le but est d'épurer le sang de certains médicaments contenus en surdose dans l'organisme et provoquant une grave intoxication (Gansè et al, 2010). L'étude expérimentale de nombreux cas d'adsorption révèle deux types d'adsorption: L'adsorption chimique et l'adsorption physique. Dans la première, les forces de liaison impliquées résultent d'un transfert ou d'une mise en commun d'électrons, alors qu'elles sont de type Van der Waals pour la deuxième. Par ailleurs, alors que l'énergie est de l'ordre $5 \mathrm{kcal} / \mathrm{mol}$ pour l'adsorption physique, elle avoisine les 150 kcals / mol pour l'adsorption chimique. L'intérêt de la molécule choisie dans cette étude réside dans ses propriétés antipaludéennes, mais surtout à cause de la surdose administrée aux malades, conséquence de la résistance du plasmodium falsipharum à ces produits. En l'absence d'un vaccin, la chimiothérapie est le seul recours contre le paludisme qui continue de se répandre dans le monde avec environ tous les ans 500 millions de nouveaux cas cliniques (Adjobimey et al., 2004). Non seulement le développement de la chimiorésistance pose un grave problème de santé publique (Cabello et al., 2011), mais ces antipaludéens exagérément absorbés provoquent de graves intoxications médicamenteuses dans l'organisme. II s'agit de trouver une technique pour, éliminer la surdose des molécules antipaludéennes de l'estomac ou de l'intestin, mais aussi épurer le sang. En effet le traitement des intoxications médicamenteuses notamment les antipaludéens nécessite non seulement un lavage gastrique mais également l'épuration du sang qui est aussi souvent intoxiqué entre la prise du médicament et le début du lavage gastrique. Notre étude est une modélisation des expériences in vitro, par l'adsorption dynamique simulant 
l'extraction des molécules médicamenteuses du sang et l'adsorption statique simulant l'extraction médicamenteuse de l'estomac. Nous avons effectué des essais d'adsorption du monohydrate du sulfate de 7- chloro-4 - (diéthylamino - 1 méthyl -1- butylamine) ou Chloroquine par différentes résines (XAD-2, XAD-4, DOWEX50W et IRA 401S) afin de déterminer celle qui a le meilleur pouvoir adsorbant vis-à-vis de la chloroquine. A

\section{MATÉRIELS ET MÉTHODES}

Matériels et produits utilisés: La Chloroquine de qualité pharmaceutique nous a été fournie par PHARMAQUICK une société de fabrication et de distribution des médicaments génériques au Bénin. Les essais ont été effectués avec deux phases stationnaires de natures différentes. Les résines $X A D-2$ et $\mathrm{XAD}_{-4}$ sont des polystyrènes fonctionnant par interactions de la liaison $\pi$ ou hydrophobe. "IRA-401"s et DOWEX 50W sont des résines échangeuses d'ions. Sur le plan de la capacité totale d'adsorption, la résine, quelle que soit sa forme ionique, présente des performances supérieures à celles de l'alumine et du silicate (Yusof et al, 2010).

Méthodes: Dans l'ensemble des expériences réalisées, nous avons mis en contact la solution à adsorber et l'adsorbant entre lesquels s'établit un équilibre; il est possible de réaliser cet équilibre de deux manières différentes sans remettre en cause le principe de base. La première consiste à immobiliser l'adsorbant dans une colonne constamment baignée par la solution étudiée - c'est l'adsorption dynamique-la seconde manière met l'adsorbant et la solution en contact dans un même récipient sous agitation, c'est l'adsorption statique (Siluestre-Albero et al , 2012).

Essais d'adsorption dynamique: La méthode utilisée est une modélisation in vitro de l'extraction des molécules médicamenteuses du sang. Elle consiste à faire circuler la solution à adsorber, représentant le sang, contenue dans un bécher de 1,5 $\mathrm{L}$ à travers une colonne garnie de résine absorbante par le moyen d'une pompe péristaltique de type GILSON Minipuls 2 et d'étudier en fonction du temps la variation de la concentration du produit en solution. Les prélèvements pour la mesure de la concentration sont de quantité suffisamment faible pour ne pas modifier de manière significative la concentration de la solution. La détermination de la concentration des solutions est obtenue par spectrophotométrie ultraviolette sur un spectrophotomètre ULTROSPEC II. partir de résines apolaires et des résines échangeuses d'ions les plus efficaces, les études cinétiques d'adsorption dynamique nous ont permis d'apprécier l'influence de divers paramètres sur le pouvoir adsorbant des résines. II s'agit de la concentration initiale de la solution, de la température, de la masse d'adsorbant, du pH et du débit.

Les courbes $C=f(t)$ obtenues traduisent un processus cinétique en rapport avec l'équilibre entre les processus d'adsorption et de désorption, car le long de la colonne s'établit un gradient de concentration. Nous exposons plus loin quelques modèles cinétiques de l'adsorption de la chloroquine; le vrai modèle est déterminé à partir de la concordance entre le calcul théorique et les résultats expérimentaux. On a envisagé les modes $[1,1] ;[1,0]$ et $[0,1]$.

Essais d'adsorption statique: Cette expérience simule l'extraction des molécules médicamenteuses de l'estomac. Dans un erlenmeyer de $500 \mathrm{ml}$, on met une masse $m$ de résine avec un volume $V$, de solution du produit à éliminer. L'ensemble plongé dans un bain thermo régularisé, est agité à vitesse constante pendant 3 heures, temps largement suffisant pour atteindre l'équilibre. Les concentrations initiales $\mathrm{C}_{0}$ et à l'équilibre $\mathrm{C}_{e}$, mesurées par spectrophotométrie ultraviolette permettent de calculer la quantité $Q_{a}$ de soluté par gramme de résine sèche selon l'expression :

$\mathrm{Qa}=\frac{(\mathrm{Co}-\mathrm{Cs}) \mathrm{Vs}}{1000 \mathrm{~m}}$

Avec $Q_{a}(g / g) ; C_{0}(g / L) ; C_{e}(g / L) ; V_{s}(m l) ; m(g)$

La désorption a été mise en évidence de la manière suivante: une masse de résine ayant adsorbé une quantité connue de soluté est séchée et remise en contact avec de l'eau pendant 3heures, sous agitation ; par dosage du produit dans l'eau, on aboutit à la quantité désorbée par gramme de résine.

Mesure de la vitesse d'adsorption: II convient de déterminer la concentration de prélèvements faits à plusieurs instants $t$ avant que l'équilibre ne soit atteint. La connaissance de $\mathrm{C}_{\mathrm{e}}$ permet de déterminer l'équilibre du processus. On parvient ainsi à calculer à chaque instant la fraction de l'équilibre obtenu à l'aide de l'expression utilisée par Bouhadiba et al, (2003) 
$\mathrm{V}_{\mathrm{a}}=\frac{C_{Q}-C_{t}}{C \theta-C \text { Eq }} * 100$

\section{RÉSULTATS ET DISCUSSIONS}

Modélisation : Au cours du processus d'adsorption l'équilibre suivant s'établit :

Produit en solution

Plusieurs auteurs ont montré que la résine jouant un rôle d'adsorbant peut être considérée soit comme un milieu homogène soit comme des grains poreux (Siluestre-Albero et al, 2012).

Comme il s'établit un gradient de concentration le long de la colonne, les diverses courbes expérimentales $\mathrm{C}=\mathrm{f}(\mathrm{t})$ traduisent un processus cinétique en rapport
Produit adsorbé sur la résine

avec l'équilibre entre les processus d'adsorption et de désorption. Bien qu'on ne parvienne pas encore à déterminer une concentration locale, il est possible de mettre en équation différents modèles.

Ainsi pour le modèle $[1,1]$, les cinétiques d'adsorption et de désorption sont toutes deux d'ordre 1 et ont les équations suivantes:

$V_{a}=\frac{d Q_{a}}{d t}=k_{a} Q_{1}-k_{-a} Q_{a} \quad$ (3) (loi cinétique) et $Q_{0}=Q_{1}+Q_{a}(4)$

Avec : $V_{a}$ vitesse d'adsorption; $Q_{a}$ quantité de produit adsorbé ; $Q_{1}$ quantité de produit en solution à chaque instant ; $Q_{0}$ quantité initiale de produit en solution; $k_{a}$ Constante de vitesse d'adsorption et $K_{-a}$ constante de vitesse de désorption

L'équation (4) dans (3) donne $\frac{d Q_{a}}{d t}+\left(k_{a}+k_{-a}\right) Q_{a}=k_{a} Q_{0}$ ainsi

$\mathrm{Q}_{\mathrm{a}}=\frac{K a}{K a+k-a} \mathrm{Q}_{0}\left[1-\mathrm{e}-\left(\mathrm{K}_{\mathrm{a}}+\mathrm{K}_{-\mathrm{a}}\right) \mathrm{t}\right]$ (5) et $\mathrm{Q}_{1}=\frac{K-a}{K a+k-a} \mathrm{Q}_{0}+\frac{K a Q_{a}}{K a+K-a} \mathrm{e}^{-(K a+k-a) t}$

Pour le modèle [1,0], la cinétique d'adsorption est d'ordre 1 et la désorption d'ordre 0 ; les équations de base sont les suivantes:

$V a=\frac{d Q_{a}}{d t}=k_{a} Q_{1}-k_{-a}$ (loi cinétique) et $Q_{0}=Q_{1}+Q_{a}$

La résolution de ces équations conduit aux expressions suivantes :

$\mathrm{Q}_{\mathrm{a}}=\frac{K a Q_{0}-K-a}{K a}\left[1-\mathrm{e}^{-K a t}\right](7)$ et $\mathrm{Q}_{1}=\frac{K-a}{K a}-\frac{K-a-K a Q_{\sigma}}{K a} \mathrm{e}^{-\mathrm{kat}}$ (8)

L'analyse du modèle $[0,1]$ où la cinétique d'adsorption est d'ordre 0 et la désorption d'ordre 1 , aboutit aux équations suivantes:

$V_{a}=\frac{d Q_{a}}{d t}=k_{a}-k_{-a} Q_{a}$ et $Q_{0}=Q_{1}+Q_{a}$ qui ont pour solution

$Q_{a}=\frac{K a}{K-a}\left(1-e^{-k a t}\right)(9)$ et $Q_{1}=\left(Q_{0}-\frac{K a}{K-a}\right)+\frac{K a}{K-a} e^{-K a t} \quad(10)$

Nous avons comparé les courbes expérimentales $C=f(t)$ obtenues au cours des essais dynamiques à celles correspondant aux différents modèles ; la meilleure concordance entre courbes expérimentales et courbes simulées pour le modèle $[1,1]$ où $Q_{1}$ est de la forme (6):

A l'équilibre c'est-à dire $t=\infty$, le deuxième terme de l'équation (6) devient nul et $Q_{1}=\frac{K a}{K a+K-a} Q_{0}(11)$ :

Sachant que $V_{a}=\frac{d Q_{a}}{d t}=k_{a} Q_{0}$. $e^{-(K a+k-a) t,}$ On en déduit que In $V_{a}=\ln k_{a} Q_{0}-\left(k_{a}+k-a\right) t$. On peut tracer la courbe correspondant à $\ln V a=f(t)$. On obtient une droite dont la pente est égale à $\left(k_{a}+k_{-a}\right)(12)$. Un programme de calculs permet donc d'obtenir les valeurs expérimentales de $k_{a}$ et $k_{-a}$ afin d'effectuer des comparaisons. Ces valeurs représentent des constantes globales apparentes caractérisant le phénomène complexe de l'adsorption

Études préliminaires : sélection de résines .Les différentes courbes expérimentales $C=f(t)$ obtenues au cours des essais préliminaires d'adsorption du sulfate de chloroquine par les différentes résines utilisées sont représentées sur la figure 1: elles montrent que les résines de types Amberlites DOWEX WH ont les meilleurs pouvoirs adsorbants. En effet le sulfate de chloroquine est une molécule polaire et, la 
résine DOWEX 50W est une échangeuses d'ions. résine DOWEX 50W.

L'étude cinétique systématique s'effectue donc avec la

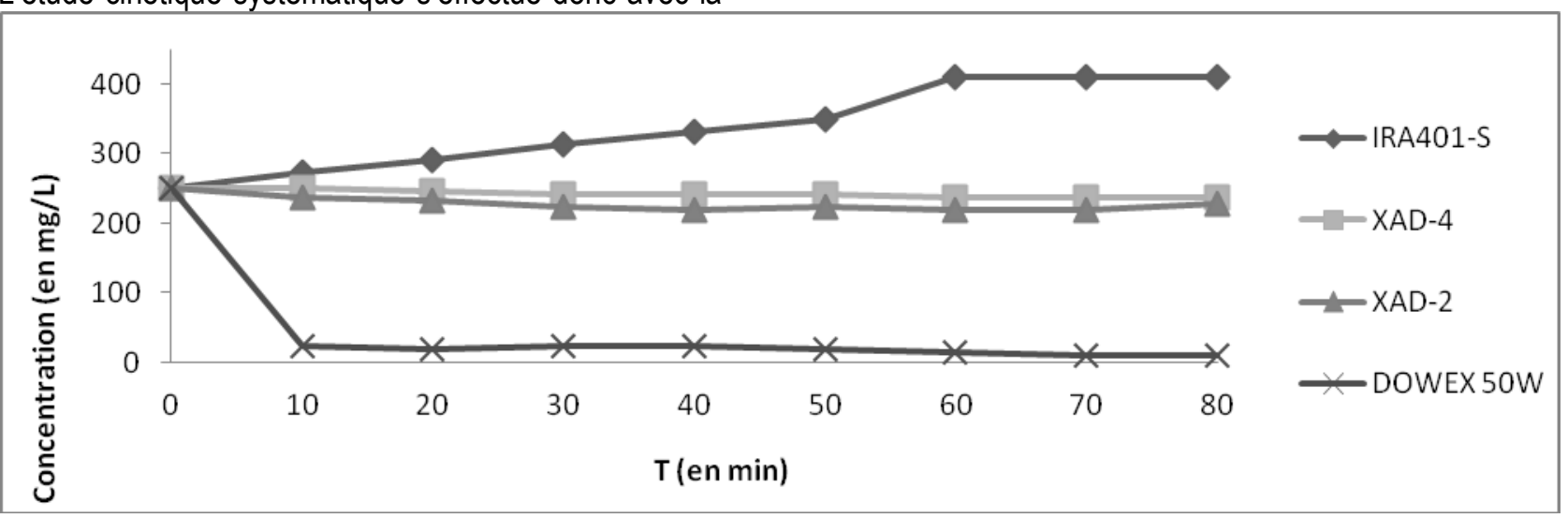

Figure 1 : Variation de l'efficacité de la résine selon sa nature

Détermination expérimentale des constantes d'adsorption et de désorption suivant les modèles cinétiques Modèles [1,1].

L'adsorption et la désorption sont toutes deux d'ordre cinétique 1. Des équations (5) et (6) qui décrivent ce modèle, on a :

$\left(Q_{1}\right)_{t=\infty}=\frac{K-a}{K a+K-a} Q_{0}$. En outre $V_{a}=\frac{d Q a}{d t}=k_{a} Q_{0} e^{-(k a+k-a) t}=\frac{C_{0}-C t}{C_{0}-C_{e q}} A v e c:$

$\mathrm{C}_{0}=$ concentration initiale; $\mathrm{C}_{\text {éq }}=$ concentration à l'équilibre; $\mathrm{C}_{\mathrm{t}}=$ concentration de la solution à l'instant $\mathrm{t}$.

$\ln V_{a}=-\left(k_{a}+k_{-a}\right) t+\ln k_{a} Q_{0}(13) ; Q_{0}$ est calculée à partir de $C_{0}$ et est équivalente à $Q_{0}=500 \mathrm{mg} / \mathrm{L}$.

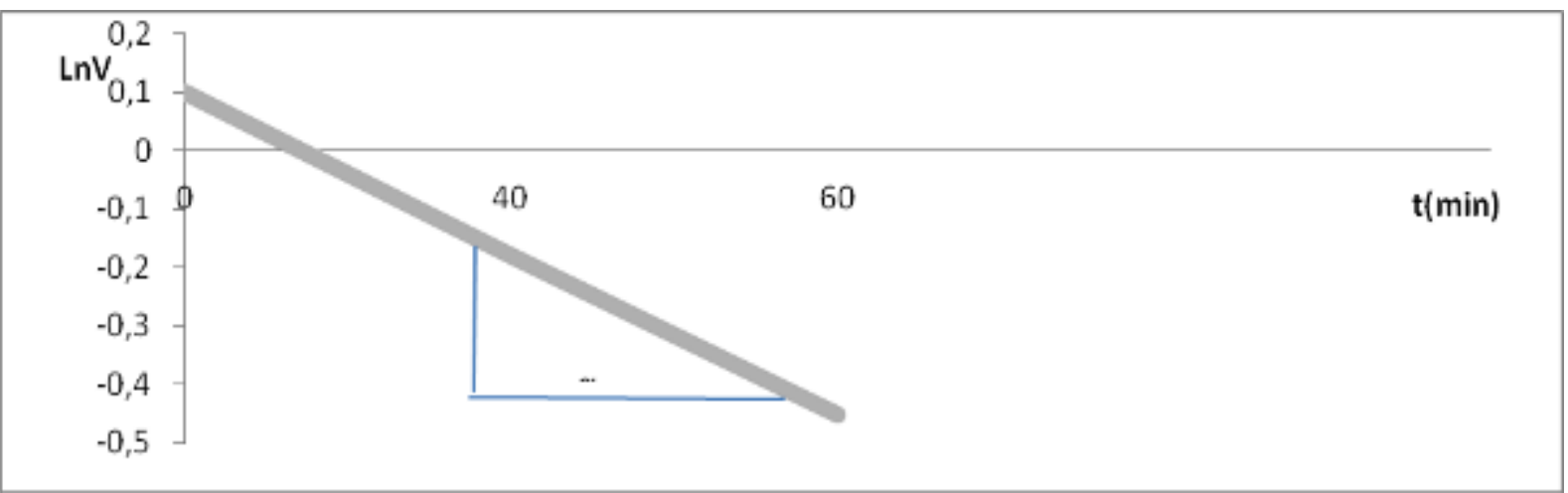

Figure 2: Variation de la vitesse en fonction du temps

A partir de la figure 2 obtenue expérimentalement, on $\mathrm{a}_{\mathrm{a}}+\mathrm{k}_{-\mathrm{a}}=\tan \alpha=8,33 \cdot 10^{-3}$

Or : $\left(Q_{1}\right) t=\infty=200 \mathrm{mg} / \mathrm{L}$ selon la figure 2 avec $\left(Q_{1}\right)_{t}$ la quantité de médicament à chaque instant $t$.

Ainsi : $\left(Q_{1}\right)_{t=\infty}=\frac{K a}{K a+K-a} Q_{0}$

$\mathrm{k}_{\mathrm{a}}+\mathrm{k}_{-\mathrm{a}}=8,33 \cdot 10^{-3}$ d'où $\mathrm{k}_{\mathrm{a}} \mathrm{Q}_{0}=200 \times 8,33 \cdot 10^{-3}$

$\mathrm{k}_{-\mathrm{a}}=\frac{200 x x_{2}, 33.10-3}{500}=3,33.10^{-3} \mathrm{~min}^{-1}$

$\mathrm{k}_{-\mathrm{a}}=3,33 \cdot 10^{-3} \mathrm{~min}^{-1}$

$\mathrm{k}_{\mathrm{a}}+\mathrm{k}_{\mathrm{a}}=8,33 \cdot 10^{-3} \Longrightarrow \mathrm{k}_{\mathrm{a}}=8,33 \cdot 10^{-3}=5 \cdot 10^{-3} \mathrm{~min}^{-1} \quad \mathrm{k}_{\mathrm{a}}=5 \cdot 10^{-3} \mathrm{~min}^{-1}$

Sachant que ce modèle est $[1,1]$ et que après calcul sur la base des données expérimentales obtenues $k_{a}>k_{-a}$ on retient la supériorité de l'adsorption.

Modèle $[1,0]$ 
L'adsorption est d'ordre cinétique 1 et la désorption est d'ordre cinétique $0 . Q_{0}=500 \mathrm{mg} / \mathrm{L}$

Ce modèle répond aux équations (7) et (8) et de ces équations on a :

$\left(Q_{1}\right)_{t=\infty}=\frac{k-a}{k a}=200$

Or $V=\frac{d Q_{a} a}{d t}=\left(k_{a} Q_{0}-k_{-a}\right) \cdot e^{-k a t}$ soit Inv=- $k_{a} t+\ln \left(k_{a} Q_{0-} k_{-a}\right)(14)$ :

D'après la figure 3 obtenue expérimentalement, on $a$ :

$\mathrm{k}_{\mathrm{a}}=\tan \beta=12 \cdot 10^{-3}$ et $\left(\mathrm{Q}_{1}\right)_{\mathrm{t}=\infty} \frac{K-a}{K a}=200 \Longrightarrow \mathrm{k}_{-\mathrm{a}}=200 \mathrm{k}_{\mathrm{a}}$ d'où $\mathrm{k}_{-\mathrm{a}}=2,4 \mathrm{mg} \cdot \mathrm{L}^{-1} \cdot \mathrm{min}^{-1}$

$k_{a}=12 \cdot 10^{-3} \mathrm{mg}^{-L^{-1}} \cdot \mathrm{min}^{-1}$ et $\mathrm{K}_{-\mathrm{a}}=2,4 \mathrm{mg} \cdot \mathrm{L}^{-1} \cdot \mathrm{min}^{-1}$

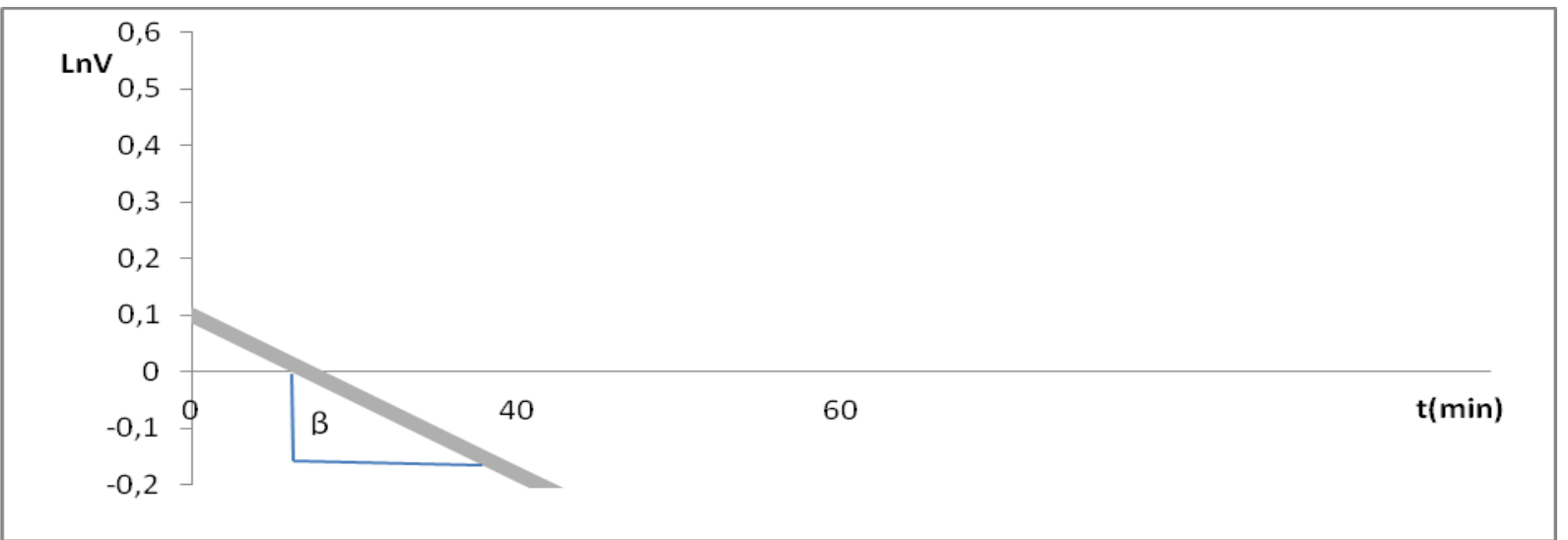

Figure 3: Variation de la vitesse en fonction du temps

Sachant que ce modèle est $[1,0]$ et que après calcul sur la base des données expérimentales obtenues $k_{-a}>k_{a}$ on retient la supériorité de la désorption sur l'adsorption donc ce modèle ne correspond pas aux résultats obtenus (voir figure1)

Modèle [0,1]

L'adsorption est d'ordre cinétique 0 et la désorption est d'ordre cinétique 1 et $Q_{0}=500 \mathrm{mg} / \mathrm{L}$

Ce modèle réponds aux équations (9) et (10): donc $\left(Q_{1}\right)_{t=\infty}=Q_{0}-\frac{k a}{k-a}=200$.

$V=\frac{d Q_{a}}{d t}=k_{a} e^{-k a t}$ d'où $\ln V=-k_{a} t+\operatorname{lnk}_{a}(15)$ :

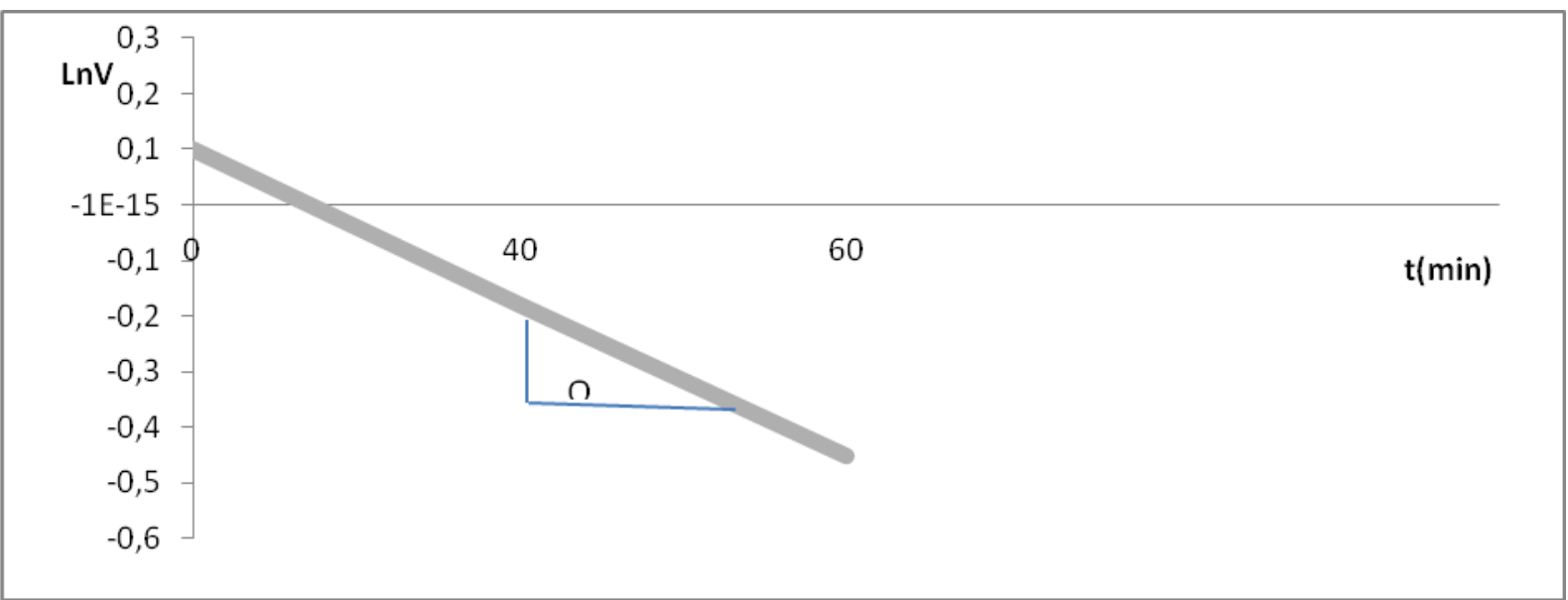

Figure 4: Variation de la vitesse en fonction du temps 
D'après la figure 4, on a:

$k_{a}=\tan \Omega=0.01 \min ^{-1}$ ainsi $\left(Q_{1}\right)_{t=\infty}=Q_{0}-\frac{k a}{k-a}=200$

$\mathrm{Q}_{0}-200=\frac{k a}{k-a}$ soit $300=\frac{k a}{k-a} \Longrightarrow \mathrm{k}_{\mathrm{a}}=300 \mathrm{k}_{-\mathrm{a}}$

$\mathrm{k}_{\mathrm{a}}=0.01 \mathrm{~min}^{-1}$ et $\mathrm{k}_{-\mathrm{a}}=3,33 \cdot 10^{-5} \mathrm{~min}^{-1}$

Sachant que ce modèle est $[0,1]$ et qu'après calcul sur la base des données expérimentales obtenues $k_{a}$ $>>k_{-a}$ on retient la grande supériorité de l'adsorption.

La constante de vitesse $k$ d'une réaction dépend de la température.

Les résultats permettent d'identifier que les modèles $[1,1]$ et $[0,1]$ sont ceux pour lesquels $K=k_{a} / k_{-a}>1$. Dans ces conditions nous pouvons donc conclure que ces deux modèles cinétiques conviennent le mieux à l'équilibre chloroquine en solution, Chloroquine retenue sur DOWEX $50 \mathrm{H}$.

Dans le cadre de la présente étude, nous avons exploité le modèle $[1,1]$. Le modèle $[0,1]$ fera l'objet d'une autre publication.

Influence de la concentration initiale: La figure 5 représente les isothermes d'adsorption de la chloroquine par la résine DOWEX. Elle montre que plus la concentration initiale est élevée, plus grande est la concentration à l'équilibre. Cette observation indique que le processus d'adsorption est soumis à un équilibre entre le produit adsorbé par la résine et sa fraction restée en solution.

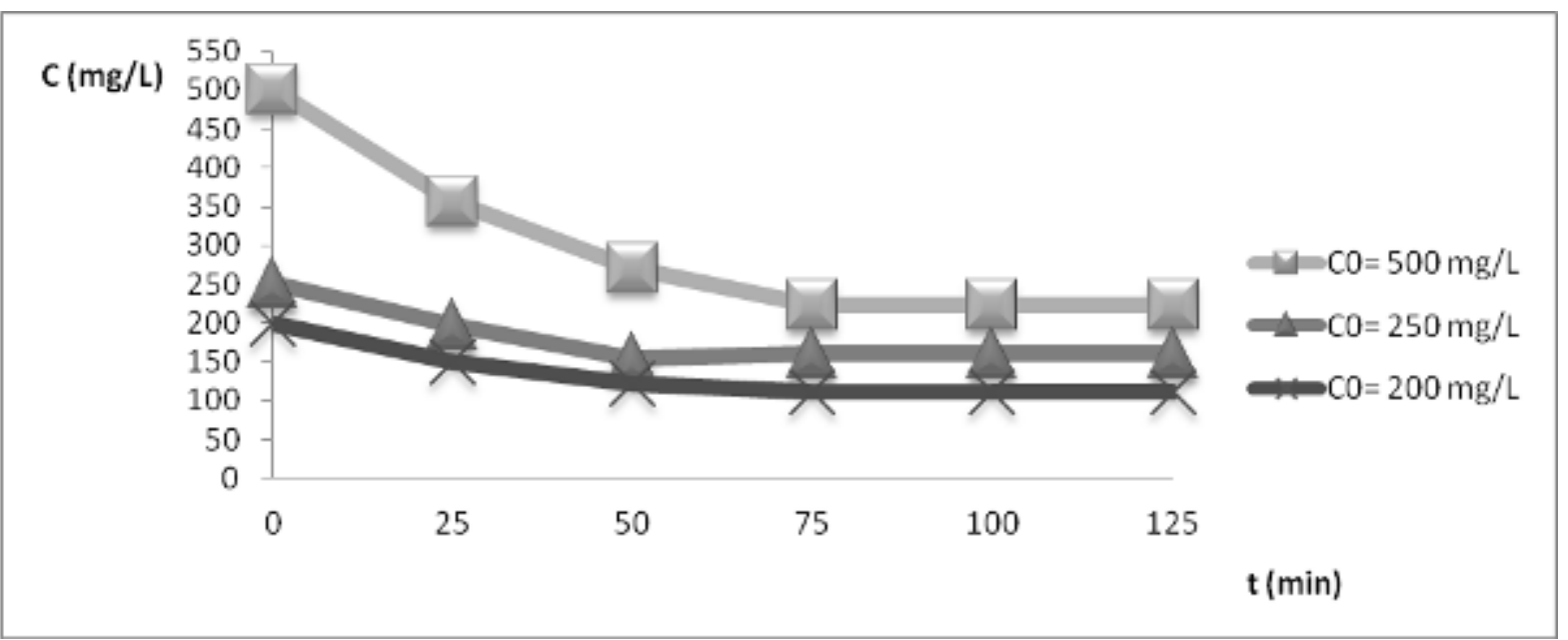

Figure 5: Variation de la concentration initiale en fonction du temps

D'après les valeurs de $k_{a}$ et $k_{-a}$ calculées à partir des figures 2,3et 4 et regroupées dans le tableau $n^{\circ} 1$ cidessous, $k_{a}$ a tendance à une légère augmentation tandis que $k_{-a}$ varie en sens inverse, ce qui a pour conséquence d'augmenter $\mathrm{K}$ avec $\mathrm{K}=\mathrm{k}_{\mathrm{a}} / \mathrm{K}_{\text {-a }}$

Tableau $n^{\circ} 1$ : Valeurs expérimentales des constantes $k_{a}$ et $k_{-a}$ en phase dynamique pour le couple chloroquine $/$ DOWEX $50 \mathrm{H}$ en fonction de la concentration initiale. $t^{\circ}=28^{\circ} \mathrm{C}$, masse d'adsorbant $=4,5 \mathrm{~g}$, débit $=20 \mathrm{ml} / \mathrm{min}$

\begin{tabular}{llll}
\hline $\mathbf{C}(\mathbf{m g} / \mathbf{L})$ & $\mathbf{k}_{\mathbf{a}}(\mathbf{m i n})$ & $\mathbf{k}_{-\mathbf{a}}(\mathbf{m i n})$ & $\mathbf{k}_{\mathrm{a}} / \mathbf{k}_{-\mathrm{a}}$ \\
\hline 200 & $3,7510^{-3}$ & $6,2510^{-3}$ & 0,6 \\
250 & $4,810^{-3}$ & $7,810^{-3}$ & 0,62 \\
500 & $510^{-3}$ & $3,3310^{-3}$ & 1,5 \\
\hline
\end{tabular}

Influence de la masse d'adsorbant: Avec l'accroissement de la masse d'adsorbant on observe une diminution de la concentration à l'équilibre (figure
6), ce qui entraîne une croissance de $Q_{a}$ Ceci se traduit par une légère croissance de la valeur de $k_{a}$ une baisse de $k_{\text {-a }}$ par conséquent $K=k_{a} / k_{-a}$ diminue (tableau ${ }^{\circ} 2$ ). 


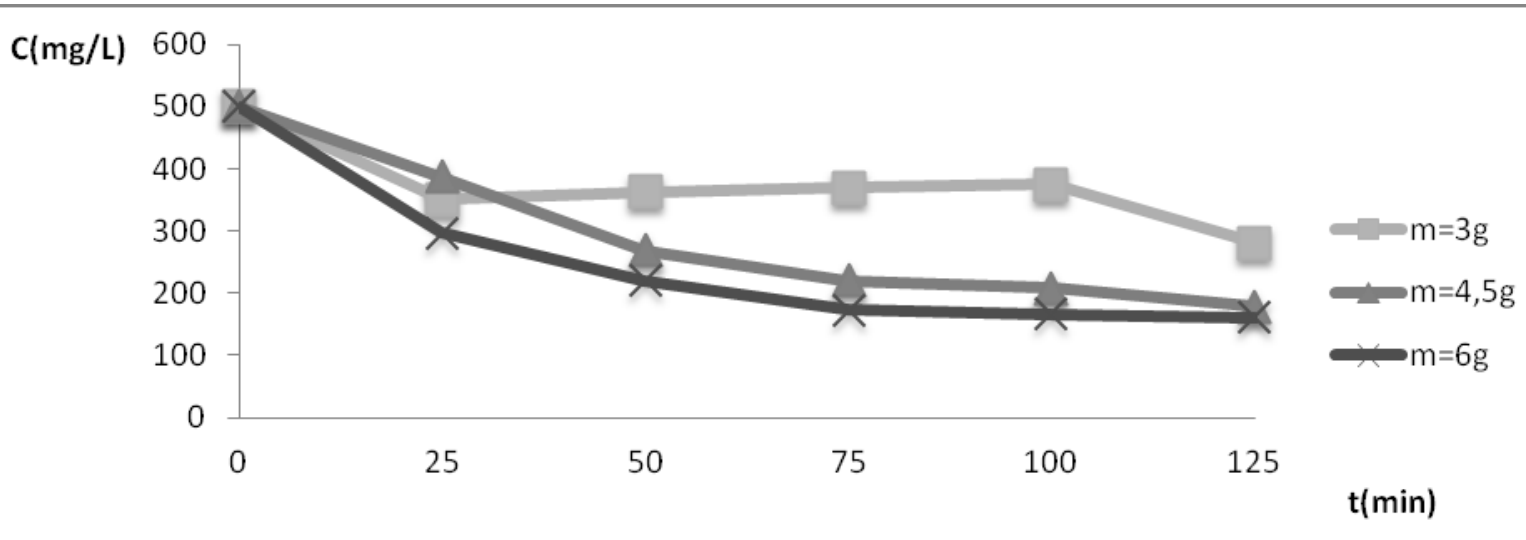

Figure 6: Influence de la masse d'adsorbant avec une concentration initiale.

Tableau 2 : Valeurs expérimentables des constantes $k_{a}$ et $k_{-a}$ en fonction de la masse pour le couple chloroquine DOWEX/50 H.

$t^{\circ}=28^{\circ} \mathrm{C}, \mathrm{C}_{0}=500 \mathrm{mg} / 1$, débit $=20 \mathrm{ml} / \mathrm{min}$

\begin{tabular}{llll}
\hline Masse de résine $(\mathbf{g})$ & $\mathbf{k}_{\mathrm{a}}(\mathbf{m i n})$ & $\mathbf{k}_{\text {-a }}(\mathbf{m i n})$ & $\mathbf{k}_{\mathrm{a}} / \mathbf{k}_{\text {-a }}$ \\
\hline 3 & $9,310^{-3}$ & $2,710^{-3}$ & 3,44 \\
4,5 & $510^{-3}$ & $3,3310^{-3}$ & 1,50 \\
6 & $5,1810^{-3}$ & $3,0410^{-3}$ & 1,70 \\
\hline
\end{tabular}

Influence du $\mathrm{pH}$ : En milieu acide $(\mathrm{pH}=3,20)$, on constate une inhibition de l'adsorption de la chloroquine d'adsorption observé avec la solution aqueuse témoin par la résine DOWEX comparativement au phénomène de chloroquine sans apport d'ions $\mathrm{H}_{3} \mathrm{O}^{+}$.

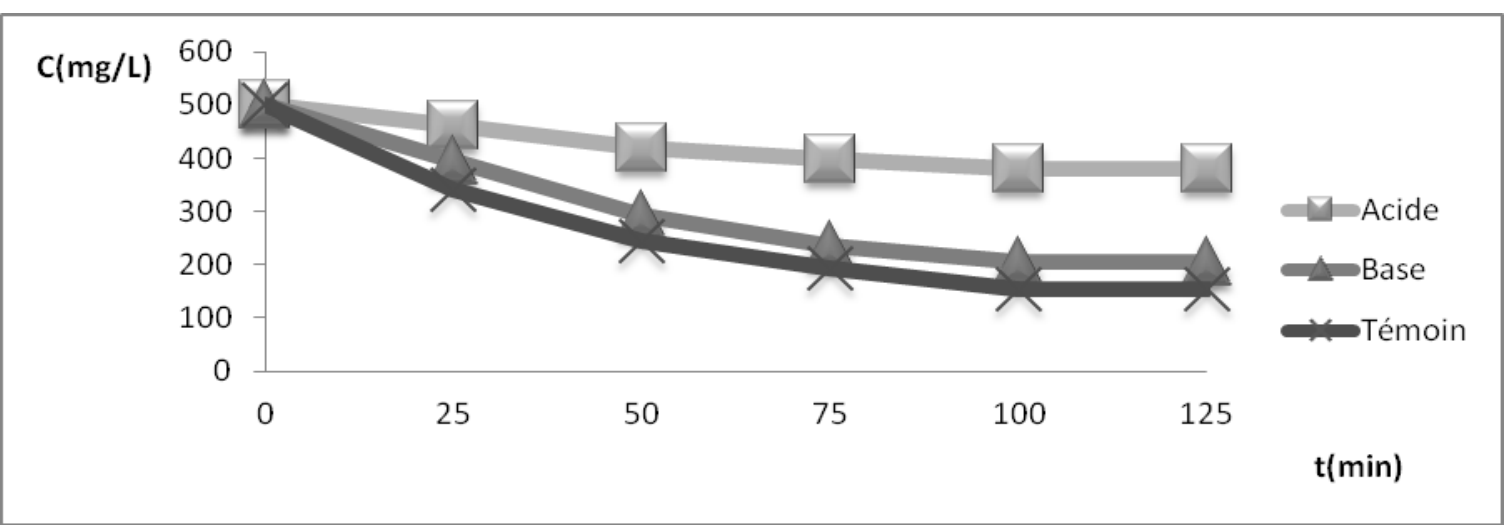

Figure 7 : Influence du pH.

En milieu basique, la chloroquine est beaucoup plus adsorbée par la résine DOWEX qu'en milieu acide et moins adsorbée qu'avec la solution témoin de

chloroquine (figure 7). En milieu acide, on remarque que $k_{a} / k_{-a}$ est inférieure à la valeur observée en milieu basique (tableau 3).

Tableau n 3: valeurs expérimentales de $\mathrm{k}_{\mathrm{a}}$ et $\mathrm{k}_{\text {-a }}$ en fonction du $\mathrm{pH}$ pour le couple chloroquine/ DOWEX 50H masse d'adsorbant : $4,5 \mathrm{~g} ; \mathrm{C}_{0}=500 \mathrm{mg} / 1$ débit $=20 \mathrm{ml} / \mathrm{min}$

\begin{tabular}{|c|c|c|c|}
\hline $\mathrm{pH}$ & $\mathrm{k}_{\mathrm{a}}(\mathrm{min})$ & $\mathrm{k}_{-\mathrm{a}}(\mathrm{min})$ & $\mathrm{k}_{\mathrm{a}} / \mathrm{k}_{-\mathrm{a}}$ \\
\hline Solution aqueuse de chloroquine+tampon acétique $\mathrm{pH}=3,20$ & $6,910^{-3}$ & $23,110^{-3}$ & 0,30 \\
\hline Solution aqueuse de chloroquine $+\mathrm{NaOH} \mathrm{pH}=10$ & $510^{-3}$ & $3,3310^{-3}$ & 1,50 \\
\hline Solution aqueuse de chloroquine. & $5,7910^{-3}$ & $4,2410^{-3}$ & 1,36 \\
\hline
\end{tabular}


Influence du débit : Quand le débit augmente, la quantité du produit absorbé à l'équilibre diminue. Le temps nécessaire pour atteindre l'équilibre augmente lorsque le débit diminue (figure.8) Lorsque le débit augmente la constante d'équilibre $\mathrm{K}$ diminue (tableau $\left.n^{\circ} 4\right)$.

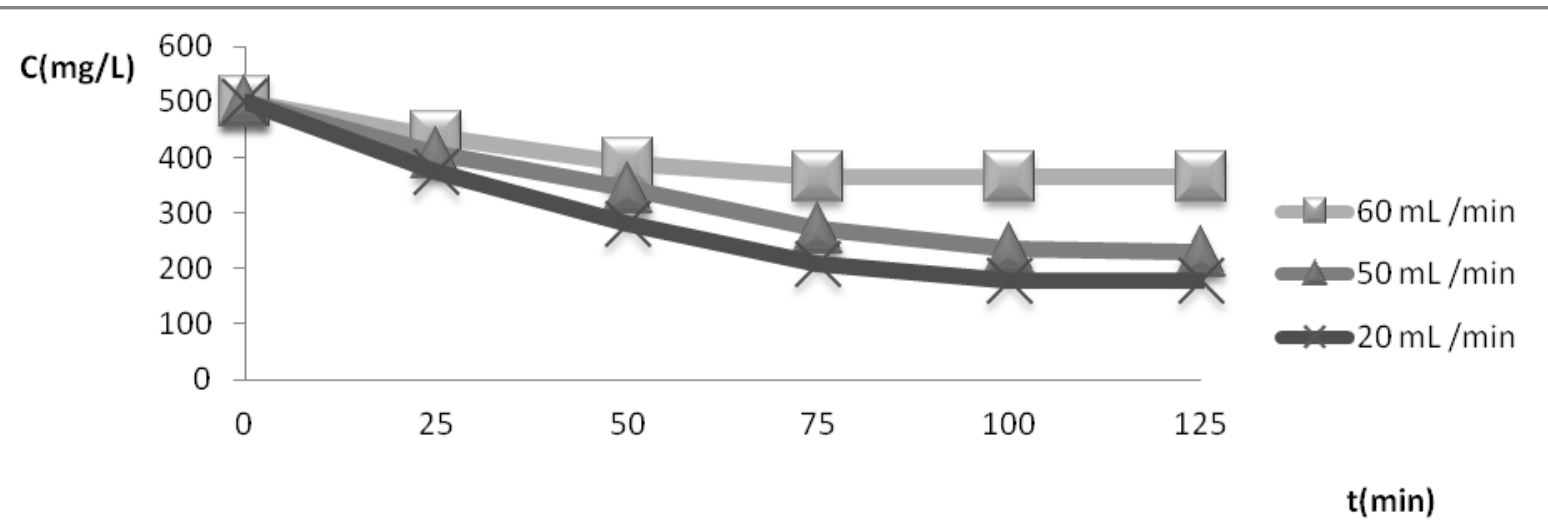

Figure 8 : Influence du débit.

Tableau 4 : Valeurs expérimentable de $\mathrm{k}_{\mathrm{a}}$ et $\mathrm{k}_{\text {-a }}$ en fonction du débit pour le couple chloroquine / DOWEX $50 \mathrm{H}$. Masse d'adsorbant : $4,5 \mathrm{~g} ; \mathrm{C}_{0}=500 \mathrm{mg} / 1 ; \mathrm{t}^{\circ}=28^{\circ} \mathrm{C}$

\begin{tabular}{llll}
\hline Débit $(\mathrm{ml} / \mathrm{min})$ & $\mathrm{k}_{\mathrm{a}}(\mathrm{min})$ & $\mathrm{k}_{-\mathrm{a}}(\min )$ & $\mathrm{k}_{\mathrm{a}} / \mathrm{k}_{-\mathrm{a}}$ \\
\hline 20 & $510^{-3}$ & $3,3310^{-3}$ & 1,5 \\
50 & $16,510^{-3}$ & $13,540^{-3}$ & 1,22 \\
60 & $1,3910^{-3}$ & $4,1610^{-3}$ & 0,34 \\
\hline
\end{tabular}

Nous remarquons que si le débit est trop grand, on obtient un mauvais contact entre les molécules et la résine. En conséquence la quantité de produit retenu sur la résine à l'équilibre est faible. On constate même que le rapport ka/k-a est très faible c'est le cas avec le débit de $60 \mathrm{ml} / \mathrm{min}$ à travers la colonne.

Influence de la température: L'augmentation de la température accroît la vitesse du processus d'adsorption, mais la quantité du produit retenu sur la résine diminue (Siluestre-Albero et al , 2012).

On observe que l'équilibre est vite atteint pour le couple adsorbat- DOWEX $50 \mathrm{H}$ (figure 9).

Plus la température augmente, plus $\mathrm{k}_{a} / \mathrm{k}_{-a}$ diminue (tableau $n^{\circ} 6$ ) $k_{a} / k_{-a}$ s'assimilant à une constante d'équilibre $K$, il s'en suit que $\ln k_{a} / k_{-a}=f(1 / T)$ est une droite de la forme $\frac{A}{T}+\mathrm{B}$.

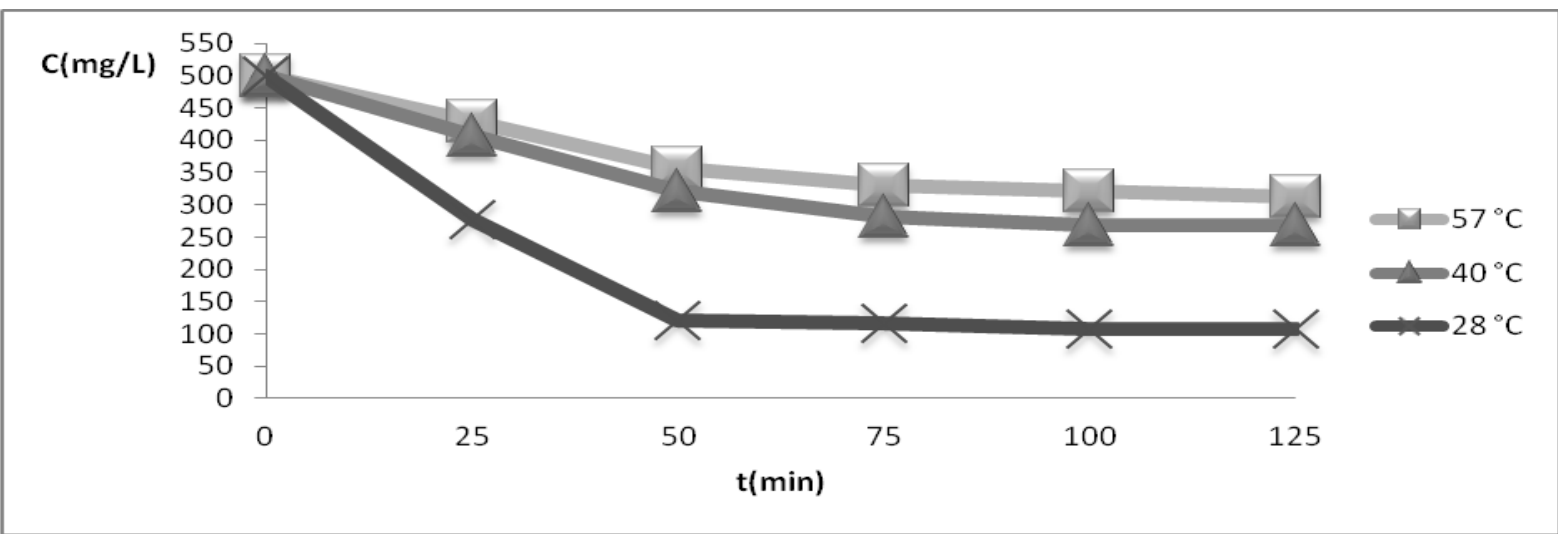

Figure 9 : Influence de la température. 
Nous savons qu'on peut l'écrire sous la forme $\operatorname{lnK}=\mathrm{A}$ $\frac{E}{R T}$ à partir de l'équation de Van'thoff où $E$ est égale à l'énergie d'activation du processus. D'après la figure 10 , nous avons $\frac{E}{R}=2976,2$ D'où $E=2976,2 \times 8,31=$ $24732,2 \mathrm{~J} \mathrm{~mol}^{-1}=24,73 \mathrm{kj} \mathrm{mol}^{-1}$ où $E=5,86 \mathrm{Kcal} / \mathrm{mol}$. Le type d'adsorption développée lors de nos mesures est probablement de la physisorption; la valeur de $\mathrm{E}$ étant légèrement supérieure à la limite de la physisorption et correspondant au début du domaine de la chimio sorption.

La variation de $\mathrm{K}$ suit donc la loi $\operatorname{lnK}=-\frac{2976,2}{T}+10$

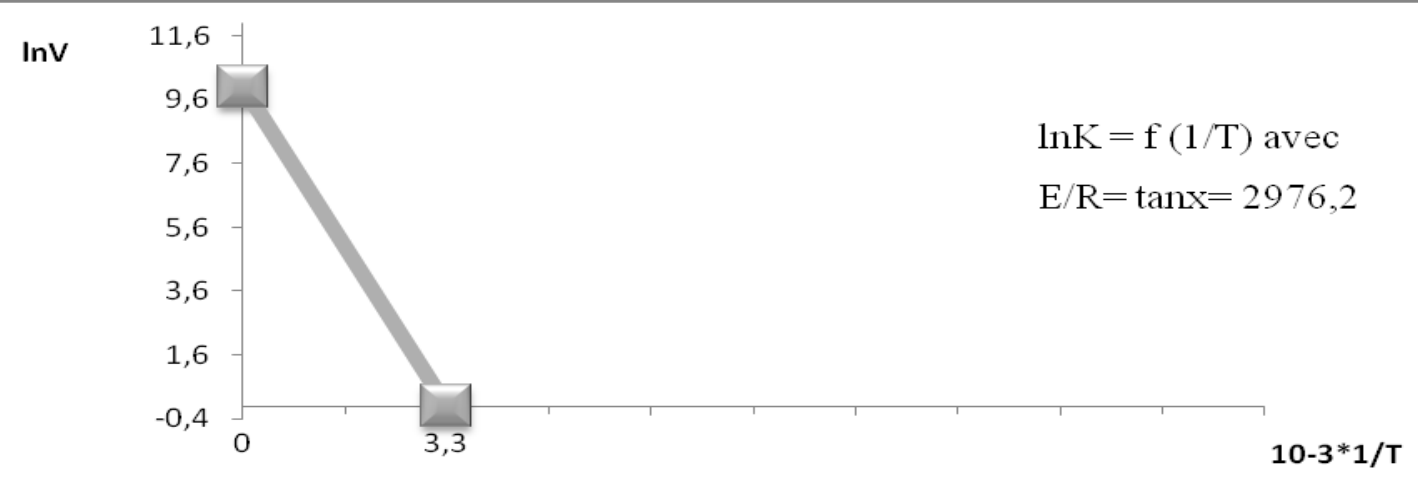

Figure $10:$ Courbe $\ln K=f(1 / T)$

Tableau 5: Valeurs expérimentales de $k_{a}$ et $k_{-a}$ en fonction de la température pour le couple chloroquine / DOWEX $50 \mathrm{H}$. Masse d'adsorbant : $4,5 \mathrm{~g}$; $\mathrm{Co}=500 \mathrm{mg} / 1$; débit $=20 \mathrm{ml} / \mathrm{min}$

\begin{tabular}{llll}
\hline Température $^{\circ} \mathbf{C}$ & $\mathbf{k}_{\mathrm{a}}(\mathbf{m i n})$ & $\mathbf{k}_{\text {-a }}(\mathbf{m i n})$ & $\mathbf{k}_{\mathbf{a}} / \mathbf{k}_{\text {-a }}$ \\
\hline 28 & $510^{-3}$ & $3,3310^{-3}$ & 1,50 \\
40 & $810^{-3}$ & $1210^{-3}$ & 0,66 \\
57 & $2,0110^{-3}$ & $5,3110^{-3}$ & 0,38 \\
\hline
\end{tabular}

\section{CONCLUSION}

Ce travail a permis de mettre en évidence que les résines utilisées présentent un pouvoir adsorbant significatif vis-à-vis de la chloroquine. Les courbes $\mathrm{C}=\mathrm{f}$ (t) des différentes résines ont permis de choisir la résine la plus efficace.

La modélisation a permis de calculer les valeurs des constantes apparentes de vitesse d'adsorption et de désorption. On peut préciser que les constantes obtenues ont un sens expérimental car leurs valeurs dépendent d'un certain nombre de paramètres. L'influence de la température sur l'équilibre a confirmé les résultats obtenus en adsorption statique, ainsi que l'influence du pH de la solution.

Les autres paramètres expérimentaux, débit à travers la colonne, masse de la résine dans la colonne, et concentration initiale, ont fait l'objet d'une étude systématique. Ce travail a permis de constater que la résine DOWEX $50 \quad(\mathrm{H})$ se prêterait mieux à l'hémoperfusion en cas d'intoxication par la chloroquine.

Bien que les expériences se soient déroulées en milieu aqueux, elles constituent une meilleure approche de l'hémoperfusion. Des études seront faites en exploitant le modèle $[0,1]$.

Compte tenu de la viscosité et des constituants du sang, cette étude s'impose afin de connaître l'influence des constituants du sang et de sa viscosité dans l'équilibre

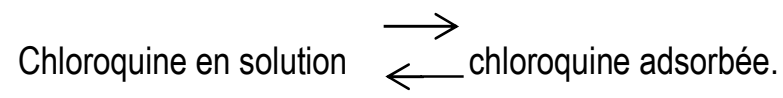

En perspective nous souhaiterions poursuivre ce travail en testant ces résines de grand pouvoir d'adsorption sur d'autres molécules médicamenteuses contenues en doses excessives dans le sang selon la nature des maladies. 


\section{BIBLIOGRAPHIE}

Adjobimey, T., Edayé, I., Lagnika, L., Gbenou, J., Moudachirou, M., Sanni, A., 2004: In vitro antiplasmodial activity of some antimalarial plants of Beninese pharmacopeia, Comptes Rendus Chimie, 7 (10-11), 1023-1027.

Bouhadiba K. and Derdour A (2003) Étude de l'adsorption de quelques benzodiazépines par des résines amberlites en milieu aquoorganique, Uni. Oran, inst. Chimie Es-senia, Algerie, Revue Journal de la Société algérienne de chimie J. Soc algér. Chim, $6 p$

Cabello, Christopher M., Lamore, Sarah D., Bair, Warner B., Qiao, Shuxi, Azimian, Sara, Lesson, Jessica L.; Wondrak, Georg T., 2011, The redox antimalarial dihydroartemisinin targets human metastatic melanoma cells but not primary melanocytes with induction of NOXA-dependent apoptosis". Investigational New Drugs $30 \quad$ (4): 1289 301.doi:10.1007/s10637-011-9676-7. PMC 3203350. PMID 21547369

Ganse H., Gbaguidi F., Agbani P., Josse R. G., Moudachirou M., Sinsin B., Quetin-leclercq J., Aminou T., 2010, Densitometric HPTLC quantification of asiaticoside isolated from Centella asiatica (L.) Urb (Apiaceae) of Benin, Int. J. Biol. Chem. Sci. 4(4): 857-863.

Karamanis D. and Vardoulakis E., 2012; Application of zeolitic materials prepared from fly ash to water vapor adsorption for solar cooling, Applied energy, pp.334-339.

Kunquan Li ; Zheng Zheng ; Xingfa Huang ; Guohua Zhao ; Jingwei Feng ; Jibiao Zhang ,2009, equilibrium, kinetic and thermodynamic studies on the adsorption of 2-nitroaniline onto activated carbon prepared from cotton stalk fibre, Journal of hazardous materials, 2009, vol. 166, n1, pp. 213-220

Shujuan Zhang, Ting Shao, Tanju Karanfil, Bingcai Pan, 2012, the correlation between structural characteristics of activated carbons and their adsorption of organic solutes from aqueous solutions, Adsorption, Volume 18, Issue 3-4, pp 229-238

Siluestre-Albero J., Silvestre-Albero A., RodriguezReinoso F., Thommes M., 2012, Physical characterization of activated carbons with narrow microporosity by nitrogen $(77.4 \mathrm{~K})$, carbon dioxide $(273 \mathrm{~K})$ and argon $(87.3 \mathrm{~K})$ adsorption in combination with immersion calorimetry, Carbon 2012, vol. 50, no9, pp. 3128-3133

Xiaomin D, Dinesh M, Charles UP J, 2013, Arsenate adsorption on three types of granular schwertmannite Original Research Article Water Research, Available online 5 February 2013

Yusof A., KEAT L. ; Zaharah I. , Majid Z. , Nizam N., 2010, Kinetic and equilibrium studies of the removal of ammonium ions from aqueous solution by rice husk ash-synthesized zeolite $Y$ and powdered and granulated forms of mordenite, Journal of hazardous materials 2010, vol. 174, n 1-3, pp. 380-385 